\title{
Analysis of 04 years (2002-2005) of laser data on Starlette, Stella and LAGEOS-I/II satellites for stations coordinates and Earth Orientation Parameters (EOP)
}

\author{
Bachir GOURINE $^{(1)}$, Kamel HASNI $^{(1)}$ and Farida BACHIR BELMEHDI ${ }^{(2)}$
}

(1) Centre of Space Techniques (CTS), Department of Space Geodesy. BP n¹3, 1 av. de la Palestine, Arzew - Algeria, Tel. +213 41793042, Fax.+213 41 792176, Email: bachirgourine@yahoo.com; bgourine@cts.asal.dz

(2) University of Oran 2, Faculty of Earth Sciences and Universe, Department of Geography, Oran - Algeria. Email : bachirbelmehdi.farida@yahoo.com

\begin{abstract}
The present paper deals with the computation of Laser stations coordinates and Earth Orientation Parameters (EOP) based on measurements of low Earth orbit (LEO) satellites, namely Starlette (STA) and Stella (STL). The orbits of these satellites are less accurate than those of the LAGEOS satellites (usually used for an accurate calculation), because they are more affected by gravitational and non-gravitational forces. The objective is to achieve a good quality on the geodetic products by inter-satellite combination of Low and High satellites data. The orbit computation of the different satellites is performed by the GINS software (GRGS/France) and the laser data processing is carried out by the MATLO software (IGN, OCA/France), considering the use of gravity field model (Eigen_Grace-03s) of GRACE satellite, over a period of 04 years (between January 2002 and December 2005). The results in terms of time series are projected onto the reference frame ITRF2000 by the CATREF software (IGN/France), where the Helmert transformation parameters are obtained. Two solutions were compared: LA1 + LA2 (LL) and LA1 + LA2 + STL + STA (LLSS), in terms of quality time series of residual positions of stations, EOP and Geocentre variations. The results show that the data obtained from LEO satellites such as Starlette and Stella can be successfully used in the accurate determination of Laser geodetic products.
\end{abstract}

Key words: Satellite Laser Ranging (SLR), Starlette, Stella, LAGEOS-I/-II, LEO, EOP, Geocentre.

\section{Introduction}

Satellite Laser Ranging (SLR) is one of the main techniques of the determination of the International Terrestrial Reference Frame (ITRF). It contributes to the frame determination by providing time series of laser stations coordinates and Earth Orientation Parameters (EOPs). The laser observations of LAGEOS-I (LA1) and LAGEOS-II (LA2) are generally used for such determination. However, what is the contribution in this determination of other satellites like Low Earth Orbit (LEO) ones?

The twins Starlette (STA) and Stella (STL) satellites, orbiting at $800 \mathrm{~km}$ altitude, were launched by the CNES, on 1975 and 1993, respectively. The main tasks of these LEO satellites are the determination of Earth's gravity field coefficients, Earth rotation parameters, and investigation of Earth and ocean tides. So, the computation of the laser ranging stations coordinates on the basis of other data than LAGEOS-I/-II (orbiting at $6000 \mathrm{~km}$ altitude) observations is desirable for the following reasons: (1) significantly increases the number of observations used for determination of the stations coordinates and EOPs, (2) verification of results obtained from the LAGEOS-I/-II data, (3) determination of station coordinates that cannot range to LAGEOS satellites. Interesting results of the stations coordinates determination were obtained for LEO satellite for short period of 01 year only [8].The objective of our study is to check if the laser ranging observations of Starlette and Stella can be used for a precise determination of the laser ranging station coordinates and EOP, and to investigate the contribution of these LEO data for the geodynamic study of the stations behaviour, pole and Geocentre motions. The work concerns the computation of a laser network, composed of 34 stations, based on both LAGEOS satellites measurements with those of Starlette and Stella over 04 years period (between January 2002 and December 2005), according to two data combination solutions, namely LA1+LA2 (LL) and LA1+LA2+STL+STA (LLSS). The methodology adopted, in this work, comprises the following steps:

a. The orbit restitution of different tracked satellites is performed by the GINS software (GRGS/ France), based on purely dynamical approach.

b. The estimation of stations coordinates updates and of EOPs residuals is performed using the MATLO software (OCA \& IGN, France) [2].This estimation provides weekly time series of stations positions and daily time series of EOPs. In order to express these parameters in same reference frame, the parameters of transformation were computed using CATREF software (IGN/France).

c. The analysis of SLR geodetic products time series based on (i) frequency analysis by FAMOUS software 
(OCA/France) [9], and (ii) noise estimation (type and level noise) by Allan variance method [4].

Finally, the preliminary results are presented and discussed.

\section{Method}

\subsection{Orbit computation}

The orbit restitution of different satellites used (LAGEOS-I, LAGEOS-II, Starlette and Stella) is performed with the GINS software, from a subset of SLR fixed stations well distributed on the Earth as reference frame for the orbitography. The quality of the positioning is directly linked to the accuracy of the orbits used (in addition to the data accuracy itself). For this reason high altitude geodetic satellites (LAGEOS-1 and LAGEOS-2) are used primarily by geodesists for SLR network computation (EOPs and stations coordinates). Indeed, these satellites have the advantage of being less sensitive to remaining uncertainties in the dynamical models than low attitude satellites like Starlette and Stella. It concerns gravitational and non gravitational effects. But since few years, global Earth gravity field models have greatly improved the accuracy of their coefficients notably thanks to the GRACE mission [10].As a consequence, empirical coefficients can be estimated along the orbit with more consistency than before; their role is to compensate part of the unknown non gravitational forces (constant and periodic). In this fact, we have used Eigen-Grace03s gravity field model for Starlette and Stella [7].

\subsection{Network and EOP computation}

The processing of SLR stations coordinates and EOP parameters was carried out by MATLO software. It comprises two main steps. The first relates to the use of the loose constraints for the resolution of the weekly normal equations systems of the network which are initially singulars due the rank defect corresponding to three rotations, in case of the Laser ranging technique. For this reason, we have applied loose constraints of about $\pm 1 \mathrm{~m}$ for stations positions and $\pm 1 \mathrm{~cm}$ for range bias per station and per satellite. The results obtained are in terms of times series of weekly coordinate updates and daily Pole parameters updates which are considered as individual solutions. Each solution generates its proper terrestrial reference frame. The second step consists in applying minimal constraints on these solutions obtained from MATLO software and to project the individual solutions according to a combined and homogeneous terrestrial reference frame using CATREF software. This projection permits to obtain the seven parameters transformation ( 3 translations, 3 rotations and 1 scale factor) between the individual solutions and the combined one. The processing results, illustrated hereafter, are expressed according to:

- parameters of transformation (translations, rotations and scale factor / ITRF2000);

- variations of pole coordinate $(X p, Y p)$ and of Length Of Day $(L O D) /$ EOPC04;

- topocentric coordinate $(N, E, U)$ updates of laser tracking stations.

\section{Results and discussion}

The SLR time series of positions expressed in the local coordinates (NEU); from the LL and LLSS combinations; are projected on ITRF2000 reference system. The results revealed that these series are statistically equivalent, according to table (1). The addition of the low satellites to the high satellites did not deteriorate the results quality, in particular for the estimation of Earth orientation and transformation parameters see table (2). In spite of the inaccuracy of the low satellites orbits (Starlette and Stella) due to the effects of the non-gravitational forces as well as the gravity field, it is now able to use them in complementary with the LAGEOS orbits; for two reasons : (a) The important quantity of the low satellites data which can contribute to well constraint the calculation of the ILRS network; (b) The good quality of the recent dynamical models (gravitational and non-gravitational) which allows an improvement of the low satellites orbits. Hereafter, we summarise some results in the following tables.

\begin{tabular}{lccc}
\hline Combination & $\begin{array}{c}\mathrm{N} \\
(\mathrm{mm})\end{array}$ & $\begin{array}{c}\mathrm{E} \\
(\mathrm{mm})\end{array}$ & $\begin{array}{c}\mathrm{U} \\
(\mathrm{mm})\end{array}$ \\
\hline LL & $-20 \pm 35$ & $21 \pm 23$ & $-6 \pm 26$ \\
LLSS & $-21 \pm 36$ & $20 \pm 21$ & $-5 \pm 28$ \\
\hline
\end{tabular}

Table 1: Average and weighted RMS of local coordinates updates of 34 Laser stations 


\begin{tabular}{lccccccccc}
\hline Solution & $\begin{array}{c}\mathrm{Xp} \\
\text { (mas })\end{array}$ & $\begin{array}{c}\text { Yp } \\
(\text { mas })\end{array}$ & $\begin{array}{c}\text { TX } \\
(\text { mm })\end{array}$ & $\begin{array}{c}\text { TY } \\
(\text { mm })\end{array}$ & $\begin{array}{c}\text { TZ } \\
(\text { mm })\end{array}$ & $\begin{array}{c}\text { RX } \\
\text { (mas })\end{array}$ & $\begin{array}{c}\text { RY } \\
\text { (mas })\end{array}$ & $\begin{array}{c}\text { RZ } \\
(\text { mas })\end{array}$ & $\begin{array}{c}\Delta \\
\left(10^{-9}\right)\end{array}$ \\
\hline LL & $-0.12 \pm 0.32$ & $0.30 \pm 0.32$ & $-1 \pm 6$ & $1 \pm 5$ & $1 \pm 7$ & $-0.13 \pm 0.41$ & $0.01 \pm 0.36$ & $-0.18 \pm 0.19$ & $-0.37 \pm 1.03$ \\
LLSS & $-0.10 \pm 0.30$ & $0.33 \pm 0.32$ & $0 \pm 6$ & $1 \pm 5$ & $1 \pm 7$ & $-0.13 \pm 0.46$ & $-0.01 \pm 0.49$ & $-0.21 \pm 0.16$ & $-0.31 \pm 0.93$ \\
\hline
\end{tabular}

Table 2: Statistics of pole coordinate updates (Xp, Yp) and the transformation parameters time series.

\subsection{Pole motion}

The figure (1) illustrates the time series residuals of pole coordinates $\left(X_{p}, Y_{p}\right)$ and of the Length of Day (LOD). These times series were processed, for LL and LLSS solutions, with respect to the standard solution EOPC04 of IERS, and expressed according to a coherent reference frame with ITRF2000. It shows that the two solutions are similar, by consequence the contribution of the Starlette and Stella data do not perturb the determination of the EOP updates. In fact, according to the table 4, the values of pole coordinates and their RMS, according to LL and LLSS solutions are practically the same. In addition, the estimation of pole parameters is satisfactory for the SLR technique and the obtained values are coherent with published values of IERS [6].
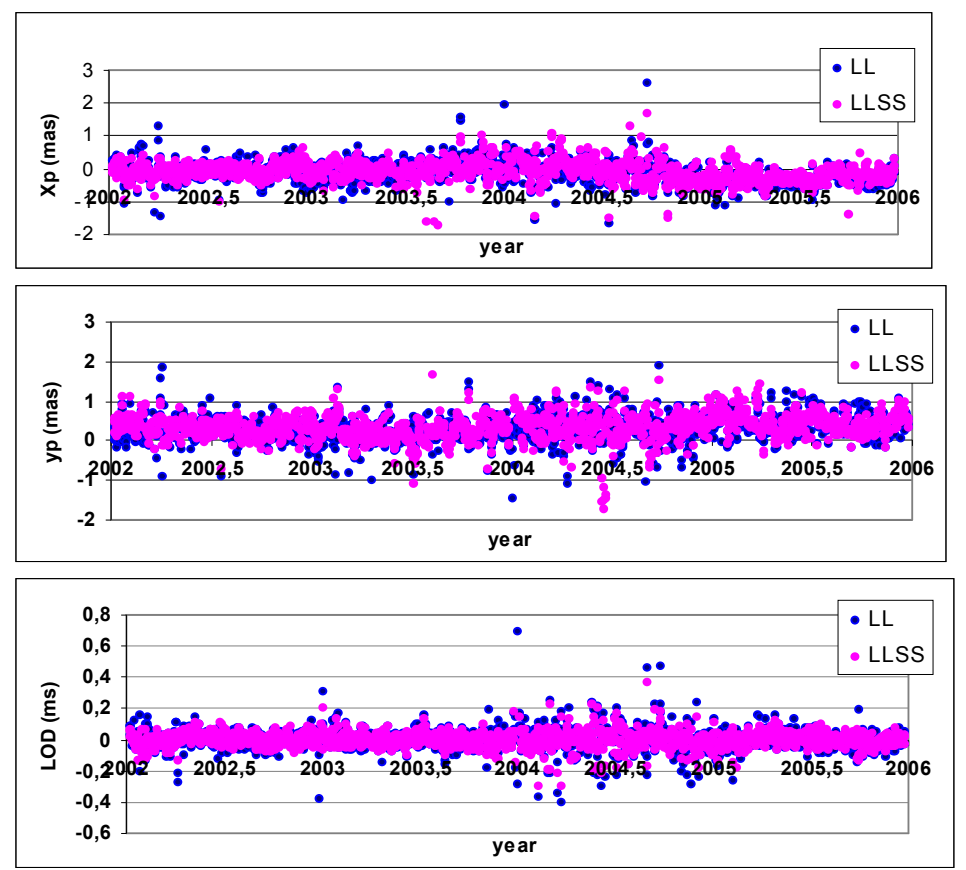

Figure 1: Parameters of pole motion according to LL and LLSS combinations.

Table (3) shows the results of frequency analysis of pole time series, using FAMOUS software. The periodic signals are decomposed, with respect to three periods: inter-annual, annual and short periods (from few days to few months $<100$ days). The choice of this decomposition is based on the periods of the geophysical phenomena causing the variations in the pole motion. These phenomena are mainly due to the redistribution of masses in the Earth, Oceans and Atmosphere [5], and were taken in account in our computations. The amplitude values of pole coordinates, according to LL and LLSS combinations, are much closed, because the maximal difference does not exceed $22 \mu$ as (i.e., $0.7 \mathrm{~mm}$ ). In other hand, the amplitudes are small of about few mm. For the LOD, the average amplitude is around $10 \mu$ s (i.e., $5 \mathrm{~mm}$ ). Generally, these values remain very small because they describe the residual signals of the geophysical phenomena.

The estimation of the noise, affecting the pole time series, has provided the level and the type of the noise. According to Figure (2), the dominant noise, for LL and LLSS solutions, is the flicker noise with a slope of the Allan diagram of -0.4 and -0.6 , respectively. The noise level is of about $106-115 \mu$ as or $3 \mathrm{~mm}$, for pole coordinates and it is around 11 and $16 \mu \mathrm{s}$ (6 and 8mm), for LOD, according to LL and LLSS, respectively. 

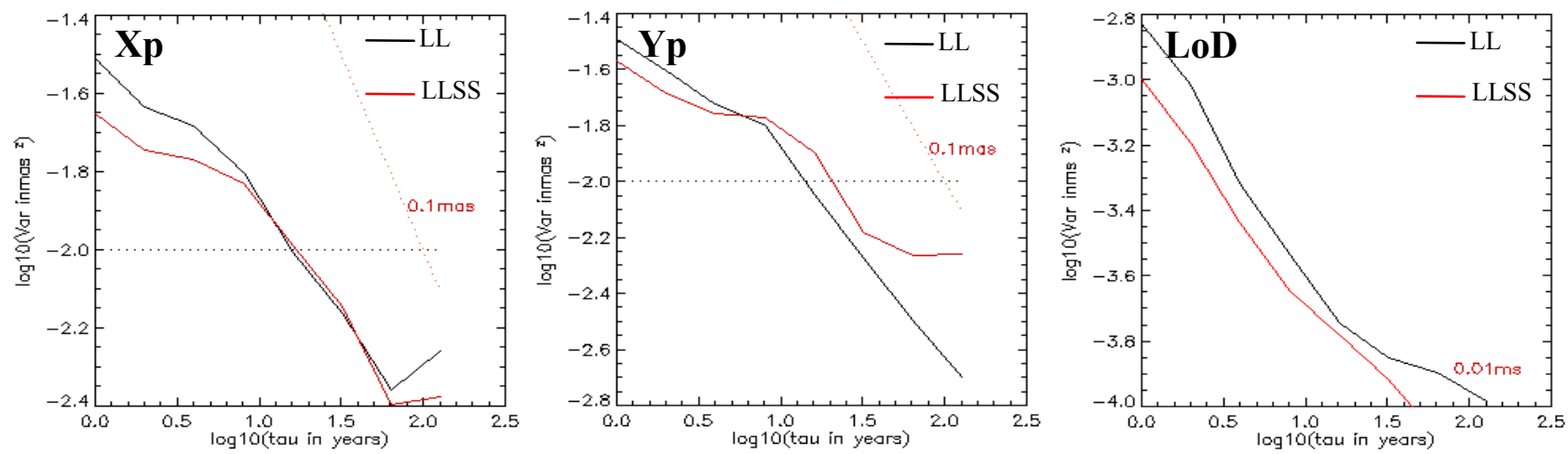

Figure 2: Allan variance diagram of the pole parameters, according to LL and LLSS solutions.

\subsection{Transformation parameters variations}

Among the weekly transformation parameters computed between the terrestrial reference frames (TRFs) and the ITRF2000, the translation parameters have a particular importance. Indeed, they permit to highlight the Geocentre variations. This is a topic of crucial importance in the Earth deformation theory as well as in the definition and maintenance of the ITRF. Figure (3) illustrates the time-series of weekly transformation parameters according to LL and LLSS solutions. The RMS estimated on the components of Geocentre are about 5-7 $\mathrm{mm}$. Usually, the variations of the reference frame scale are affected by the determination errors of stations vertical coordinates [2]. So, range biases and errors of the radial components due to the orbit residual errors affect also the scale variations. According to the table (4), the results about the scale factor are at the same level for the two solutions (LL and LLSS). The average RMS is about $6 \mathrm{~mm}$ for both solutions.
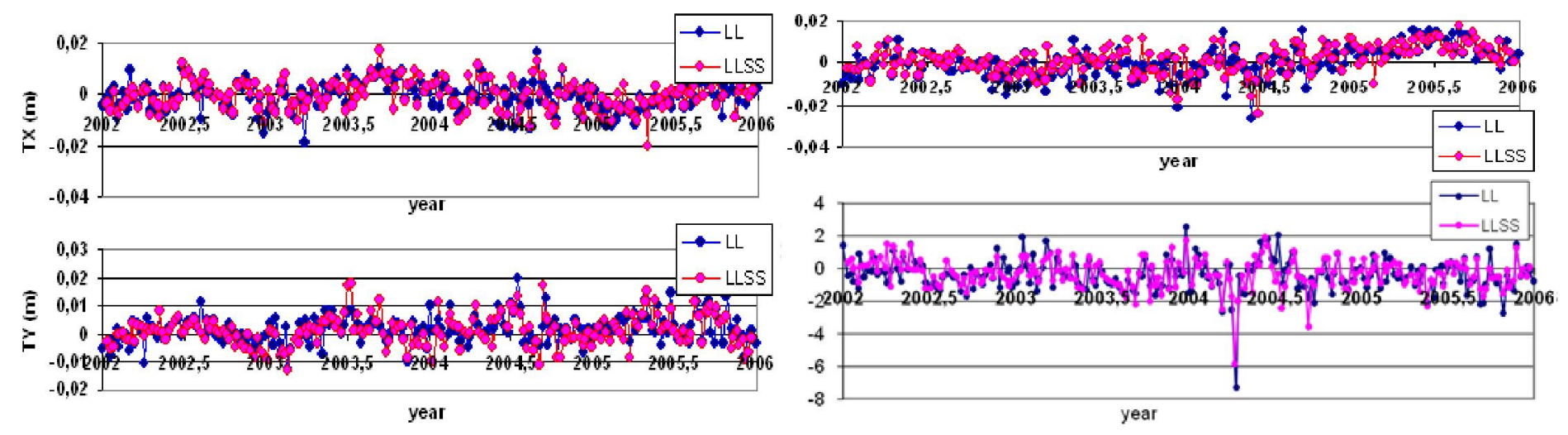

Figure 3: Time series of translations and scale factor variations, according to LL and LLSS combinations.

The Geocentre variations are mainly due to the redistribution of masses in atmosphere, in oceans and also in hydrological reservoirs. In general, they present two principal periodic components: annual and semi-annual terms. Table (3) displays the values of amplitudes and phases of annual terms of our solutions, and of two geodynamic models of [1-3], computed taking into account atmospheric pressure data, ocean tides and surface water data. We can observe equivalent amplitude values of TX and TZ, for the LL and LLSS solutions. There is coherence in amplitude between our solutions and geodynamical models, for TX and TY. However, a difference of about $1-2 \mathrm{~mm}$ exists for TZ component.

\begin{tabular}{|r|c|c|c|c|}
\cline { 2 - 5 } \multicolumn{1}{c|}{} & \multicolumn{1}{c|}{ LL } & LLSS & \begin{tabular}{c} 
Dong et al. 1997 \\
\multicolumn{1}{c|}{}
\end{tabular} & $\begin{array}{c}\text { Chen et al. 1999 } \\
{[1]}\end{array}$ \\
\hline TX A $(\mathrm{mm})$ & $2.9 \pm 0.8$ & $2.6 \pm 0.8$ & 4.2 & 2.4 \\
$\varphi\left({ }^{\circ}\right)$ & $139 \pm 15$ & $131 \pm 18$ & 224 & 244 \\
\hline TY A $(\mathrm{mm})$ & $2.3 \pm 0.5$ & $4.1 \pm 0.6$ & 3.2 & 2.0 \\
$\varphi\left({ }^{\circ}\right)$ & $168 \pm 22$ & $183 \pm 16$ & 339 & 270 \\
\hline $\mathrm{TZ} \mathrm{A}(\mathrm{mm})$ & $2.3 \pm 2.6$ & $1.9 \pm 2.1$ & 3.5 & 4.1 \\
$\varphi\left({ }^{\circ}\right)$ & $246 \pm 67$ & $218 \pm 71$ & 235 & 228 \\
\hline
\end{tabular}

Table 3: Annual terms of the Geocentre variations components according to LL and LLSS combinations. 
The spectral behaviour of the Geocentre motion, described by Allan variance method, is illustrated by the figure (4). The white noise is the dominant noise for the X and Y Geocentre components, with noise level of about 1.8 $\mathrm{mm}$ (according to the LL and LLSS combination but it is about $2.3 \mathrm{~mm}$ for Y-component of LLSS solution). However, the Z-component is affected by a flicker noise at level of $2.8 \mathrm{~mm}$.
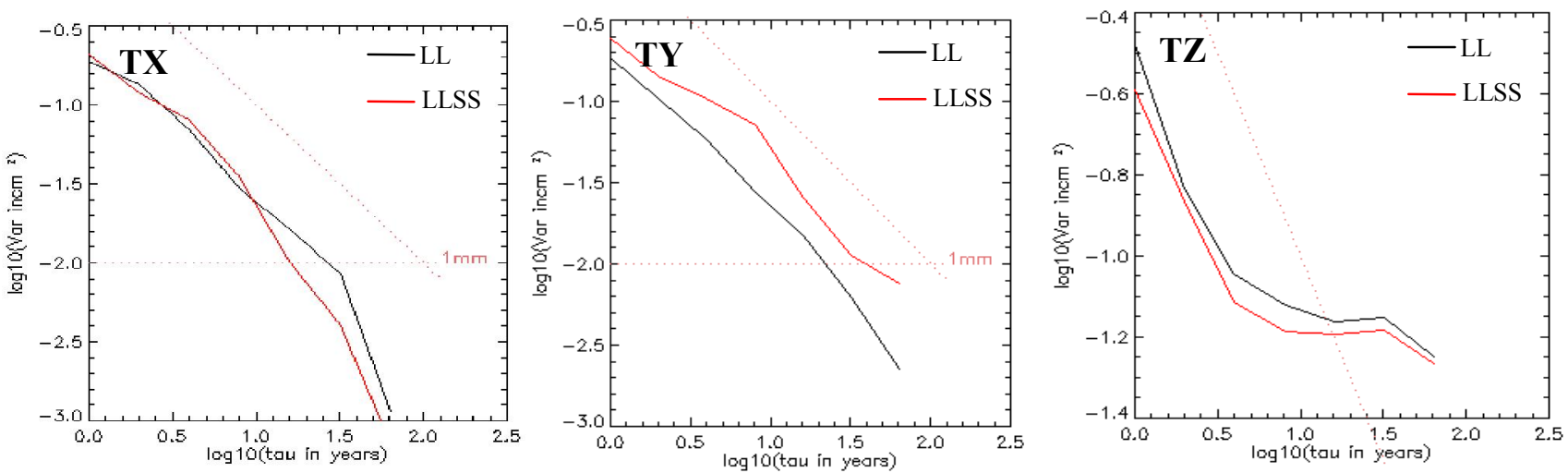

Figure 4: Allan variance diagram of the Geocentre variations, according to LL and LLSS solutions.

\subsection{Coordinate updates of SLR stations}

Figure (5) shows the geographical distribution of the 34 SLR stations considered in this study.

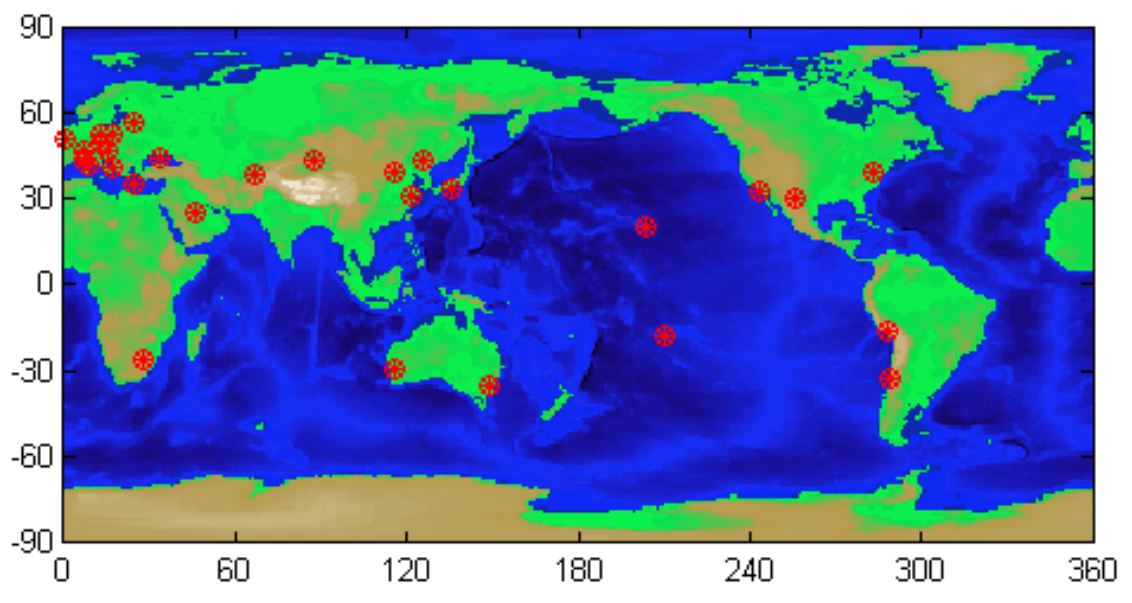

Figure 5: SLR Network considered in this study.

The following figure shows the average RMS of topocentric coordinates (North, East and UP) of 34 SLR Stations over four years (2002-2005), according to the LL and LLSS combination solutions.

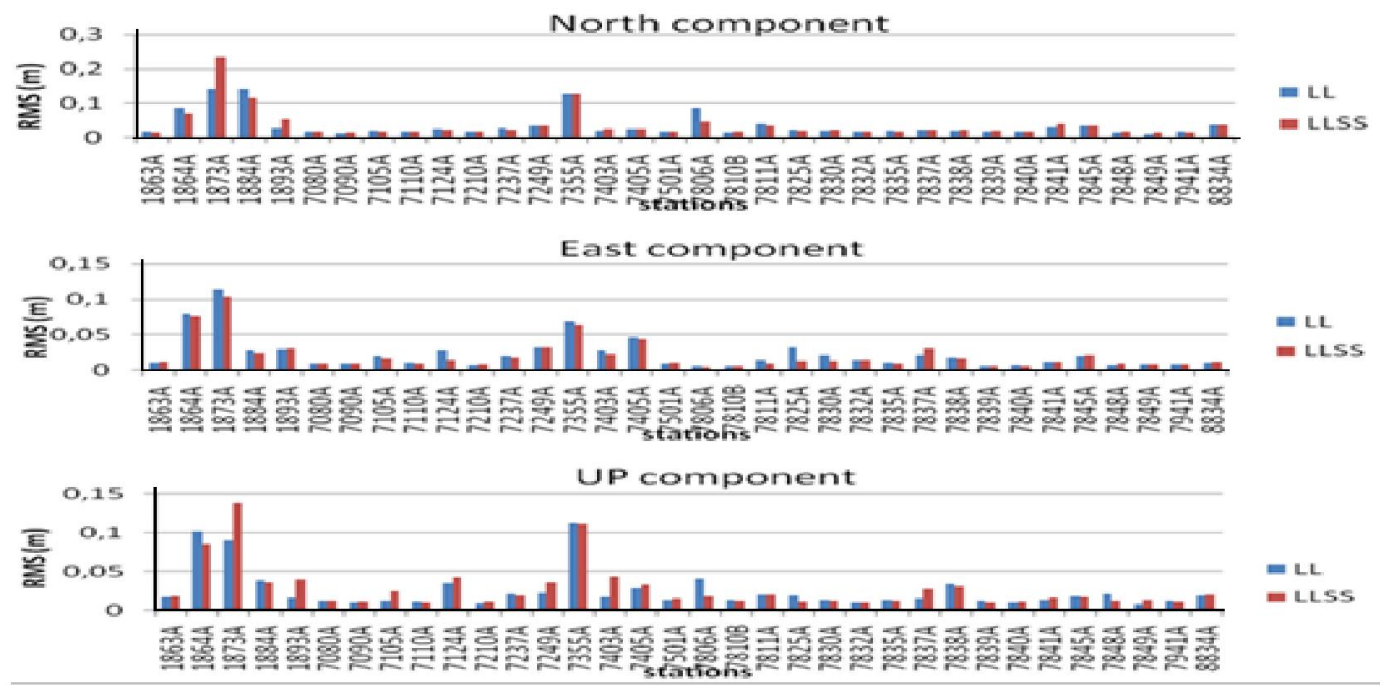

Figure 6: RMS of topocentric coordinates of 34 SLR stations, according to LL and LLSS solutions. 
The interest of the time series of the stations coordinates calculated in a homogeneous reference frame is to enable us to highlight residual signals compared to the a priori signals used in modelling (geophysical signals). We focused on vertical component because it is important for the geodynamical studies since it holds amplitude $2 / 3$ of signals acting on the station motion [2].In this context, one carried out a frequency analysis on vertical component series by FAMOUS software. Seasonal signals with amplitudes of about few $\mathrm{mm}$ were estimated. Since, the effects of ocean loading were considered in the model a priori of restitution, the signals detected are probably related to residual loading effects, which typically have amplitudes of mm level.

\section{CONCLUSION}

This study showed that good quality on SLR geodetic products (stations coordinates, EOP, Geocentre) can be achieved by inter-satellite data combination of LEO satellite such as Starlette and Stella with high satellite as LAGEOS1 \& LAGEOS2. The positions of 35 stations are estimated with the same RMS of about $3 \mathrm{~cm}, 2 \mathrm{~cm}$ and $3 \mathrm{~cm}$, for local coordinates (N, E, U), respectively, considering the two solutions (LL and LLSS combinations). The analysis methodology, developed in this context, which is based on the frequency analysis by FAMOUS software and noise estimation by Allan variance method, revealed that for both solutions : (i) the quality on EOP time series is the same and coherent with published values of IERS [6]. Small amplitudes were estimated on pole coordinates updates (Xp, Yp) and LOD. The flicker noise is the characteristic noise of the EOP with level of about $3 \mathrm{~mm}$ level for pole coordinates and $8 \mathrm{~mm}$ for LOD. (ii) the amplitudes of annual signals estimated on Geocentre variations are equivalents and are coherent with geodynamic models ones. The white noise is the dominant noise for the TX and TY components at level of about $2 \mathrm{~mm}$; however, the TZ component is affected by a flicker noise at level of $3 \mathrm{~mm}$. For extending this work, it is interesting, to consider more observations of LEO satellites (such as, Ajisai, TopexPoseidon, Jason-1\&-2, with Starlette and Stella), during a long period.

\section{Acknowledgements}

The author thanks Prof. Exertier and his research team (GMC/GEMINI/OCA-France) for their scientific and technical support, and their help for the use of GINS, MATLO, FAMOUS and CATREF programs.

\section{References}

[1] Chen, J. L., Wilson, C. R., Eanes, R. J. \& Nerem, R. S, 1999: Geophysical interpretation of observed geocenter variations. Journal of Geophysical Research, 104(B2) : 2683-2690.

[2] Coulot, D., Télémétrie laser sur satellites et combinaison de techniques géodésiques. Contributions aux systèmes de référence terrestres et applications. PhD thesis (in French), Observatoire de Paris, 2005.

[3] Dong, D., Dickey, J. O., Chao, Y \& Cheng, K., 1997: Geocenter variations caused by atmosphere, ocean and surface ground water. Geophysical Research Letters, 24(15) : 1867-1870.

[4] Feissel-Vernier, M., de Viron, O., Lebail, K., 2007: Stability of VLBI, SLR, DORIS, and GPS positioning. Earth Planets Space, 59, 475-497.

[5] Frède, V., 1999: Apport de l'analyse non linéaire à l'étude géophysique de la rotation de la Terre. Thèse de doctorat, Observatoire de Paris, France.

[6] Gambis, D., 2004: Monitoring earth orientation using space-geodetic techniques: state-of-the-art and prospective. Journal of Geodesy, 78: 295-305.

[7] Gourine, B., S. Kahlouche, P. Exertier, Ph. Berio, D. Coulot, and P. Bonnefond, 2008: Corsica SLR positioning campaigns (2002 an 2005) for satellite altimeter calibration missions. Marine Geodesy, 31: 103-116, doi: $10.1080 / 01490410802053658$.

[8] Lejba, P., Schillak, S., Wnuk, E., 2007: Determination of orbits and SLR stations' coordinates on the basis of laser observations of the satellites Starlette and Stella, Advances in Space Research, Volume 40, Issue 1, p.143-149.

[9] Mignard, F., 2005: Guide d'utilisation du logiciel FAMOUS. OCA Internal Report (in French) - France.

[10] Reigber Ch., R. Schmidt, F. Flechtner, R. Konig, U. Meyer, 2005: An earth gravity field model complete to degree and order 150 from GRACE: Eigen-Grace02S. Journal of Geodynamics, Vol. 39, 1-10. 